\title{
Second-order conditioning using a contextual stimulus as $\mathbf{S}_{1}$
}

\author{
NANCY A. MARLIN \\ University of Missouri, Rolla, Missouri
}

\begin{abstract}
Three experiments investigated the question of whether a spatial stimulus, a context, could function as $S_{1}$ in a second-order conditioning procedure. In each experiment, rat subjects were presented with $S_{1}$-US pairings by being given footshocks in one of two contexts. Forty-eight hours later, the experimental groups received $S_{2}-S_{1}$ pairings, during which a tone was presented in the training context. As measured by a lick-suppression test administered in a third context, rats were more fearful of the tone if it occurred in the context in which they had previously been shocked. The training context in each experiment apparently served to establish second-order fear conditioning to the tone.
\end{abstract}

There has been an increasing amount of research investigating the role of contextual or background cues in conditioning processes. The majority of this research has focused on first-order conditioning within a theoretical framework that views the context as a competing element of a compound conditioned stimulus (cf. Rescorla \& Wagner, 1972). The appropriateness of this viewpoint is supported by analyses of contextual conditioning in such phenomena as blocking (Balsam \& Schwartz, 1981; Tomie, Murphy, Fath, \& Jackson, 1980) and overshadowing (Marlin, 1981; Odling-Smee, 1978). These findings encourage the interpretation that the context, in at least some circumstances, does appear to function as a conditioned stimulus (CS).

One property acquired by a CS during Pavlovian conditioning is the ability to subsequently serve as a reinforcer for another stimulus. Therefore, if the context functions as a CS, it ought to be possible to use a conditioned context as a reinforcer in a secondorder conditioning procedure. The actual design of such a procedure would appear to be relatively straightforward: one stimulus, in this case a context, would be paired with an unconditioned stimulus (US), and then a second stimulus would be paired with this context. The association of concern is whether $S_{2}$, the second stimulus, is conditioned as a result of its pairing with $S_{1}$, the context.

Although this procedure conforms operationally to second-order conditioning, the unique characteristics of contextual cues need to be considered in such

This research was supported by National Institute of Mental Health Grant MH36914-01. I wish to thank Kim Oostendorp, Kathryn Bushur, and Charles Lipscomb for their assistance in the data collection. Reprints may be obtained from Nancy $A$. Marlin, Department of Psychology, University of Missouri, Rolla, Missouri 65401. a design. Even ignoring the theoretical role of the context, it is clear that it empirically differs from stimuli typically used as $S_{1}$ in second-order conditioning. An obvious difference is that a context is constantly present throughout the experimental session. Even though a US may occur within the context and the contextual stimulus may be the best predictor of the US occurrence, this relationship is not well described by traditional temporal categories such as forward or backward conditioning. Of course, it is possible to arrange these types of temporal pairings with contextual stimuli. For example, Best, Best, and Mickley (1973) used forward pairings of a taste with a context to demonstrate second-order taste aversion conditioning to an exteroceptive stimulus by presenting subjects with a saccharin solution prior to placing them in a black compartment.

More commonly, however, pairing a context with a US or pairing an $S_{2}$ with a context actually involves embedding the US or $S_{2}$ within the target context. This is because the external context, as contrasted with contextual stimuli such as motivational states or drug-induced cues (e.g., Cunningham, 1979), is essentially a spatially defined rather than a temporally defined stimulus. Although all stimuli can be considered to possess both spatial and temporal characteristics, the relative duration of the stimulus may influence whether its spatial or its temporal properties are more salient. If, for example, a white noise was presented for a few seconds, its temporal characteristics would probably be most obvious. Yet, if this same white noise was present throughout the experimental session, its spatial characteristics would likely predominate and it would be best described as a background, apparatus, or contextual stimulus. The following experiments investigated whether such a spatial stimulus could serve as an $S_{1}$ in secondorder conditioning. 


\section{EXPERIMENT 1}

To demonstrate second-order conditioning, it is necessary to show that the conditioned response to $S_{2}$ depends on both its pairing with $S_{1}$ and the prior pairing of $S_{1}$ with a US. In Experiment 1, all subjects received shock within a particular context $\left(\mathrm{S}_{1}\right)$. Fortyeight hours later, half of the subjects received presentations of a tone $\left(S_{2}\right)$ within the conditioned context; the remaining half of the subjects received this tone in a different context. All animals were then tested for their conditioned responses to the tone in a third context. If the observed response to the tone was specific to the $S_{1}-U S$ and $S_{2}-S_{1}$ pairings, then the animals receiving the tone in the conditioned context would be predicted to be more fearful of the tone than would those animals receiving the tone in a different context.

\section{Method}

Subjects. Eight experimentally naive Sprague-Dawley-derived adult female rats (Charles River Co.), weighing between 255 and $310 \mathrm{~g}$, served as subjects. They were individually housed in wiremesh cases in a colony room maintained on a $12 / 12$-h light-dark cycle. They were maintained on an ad-lib Purina Laboratory Chow diet and a 23.5-h water-deprivation schedule. The animals were placed on this water-deprivation schedule 2 days before the beginning of the experiment. During the study they were given water approximately $1 \mathrm{~h}$ after the completion of the experimental treatment. All subjects had been adapted to handling prior to the study.

Apparatus. $S_{1}-$ US pairings for all animals occurred in one of two distinct contexts in different rooms of the laboratory. One apparatus was a $30 \times 10 \times 15 \mathrm{~cm}$ compartment with a floor made of $.64-\mathrm{cm}$-wide metal grids spaced $1.27 \mathrm{~cm}$ apart. Each pair of adjacent metal grids was connected by an NE-2H neon bulb, such that each grid could be electrified with respect to every other grid by the $5,000-\mathrm{V}$ shock source. The walls of this apparatus were black Plexiglas, and the ceiling was clear Plexiglas that was perforated to allow for the tone delivery. The room in which this apparatus was housed was dimly illuminated, and $7 \mathrm{ml}$ of peppermint extract was uniformly spread on the bedding beneath the apparatus.

The second apparatus had a floor that was $25.4 \mathrm{~cm}$ in length and was made of two parallel stainless steel plates, each $3.2 \mathrm{~cm}$ wide, with a $1.3-\mathrm{cm}$ gap between them that could be bridged by the animal. The side walls were made of these same plates, which angled outward equally from the floor to a maximum width of $21.6 \mathrm{~cm}$ at the $24.1-\mathrm{cm}$-high ceiling. Each plate was connected to one pole of the shock source. The two end walls were covered with 2.5 -cm-wide vertical black and white stripes, a florescent bulb was mounted above the clear Plexiglas ceiling, and $7 \mathrm{ml}$ of lemon extract was spread on the bedding beneath the apparatus.

A third apparatus was used to measure lick suppression to the tonal $\mathrm{S}_{2}$. This lick apparatus was a $30 \times 25 \times 23 \mathrm{~cm}$ clear Plexiglas enclosure with a floor of $0.32-\mathrm{cm}$-wide metal grids spaced $1.27 \mathrm{~cm}$ apart. A circular opening, $1 \mathrm{~cm}$ in diameter, was centered in a side wall $1.9 \mathrm{~cm}$ above the floor. A water tube connected to a lickometer circuit protruded $1 \mathrm{~cm}$ beyond the side wall through this opening. The lick apparatus was enclosed in a sound-attenuating chamber.

Procedure. The experiment was conducted over 4 consecutive days; all procedures occurred at approximately the same time of day, during the light portion of the dark/light cycle. On Day 1, each water-deprived subject was placed in the center of the lick apparatus and was allowed to explore and find the lick tube. Three minutes after its first lick, the subject was removed from the ap- paratus and returned to its home cage. Day 2 consisted of the first phase of second-order conditioning. Each subject was placed in one of the two training contexts and given four .5-sec, .5-mA constant-current footshocks with a mean intershock interval of $75 \mathrm{sec}$. The actual assignment of the training context was counterbalanced across subjects. On Day 3, each subject was given another exposure to the lick apparatus. This was identical to the exposure given on Day 1 and served to reestablish the subjects' baseline drinking rates. Day 4 was the second phase of second-order conditioning. All subjects received two presentations of a 5-sec, 1600 $\mathrm{Hz}, 77-\mathrm{dBA}$ (re $20 \mu \mathrm{N} / \mathrm{m}^{2}$ ) tone. For half of the animals within each training context, this tone was paired with the context in which they had received the footshocks $\left(S_{2}-S_{1}\right)$; for the remaining half of the subjects, the tone was paired with the different context. Beginning $30 \mathrm{~min}$ after these pairings, all animals were tested for their fear of the tone $\left(S_{2}\right)$. For this test, each subject was placed in the lick apparatus. After the subject's fifth lick, the tone was presented and left on continuously until the subject had completed 40 licks. The latency to begin licking and the latency from Lick 5 to Lick 40 , in the presence of the tone, were recorded. A 900 -sec ceiling score was adopted.

\section{Results and Discussion}

The data were analyzed by comparing Group Same, the subjects that had received the tone in the context that had previously been paired with the shock, with Group Different, the subjects that had received the tones in the context that had not been paired with the shocks. All lick latencies were transformed to $\log$ seconds (base 10) to normalize their distributions; two-tailed rejection regions were used for all statistical tests. On Days 1 and 3, there were no reliable differences between the two groups in their latencies to begin licking or in the total number of licks made during the 3-min lick interval (all ps > .05 ). During the lick suppression test on Day 4, the two groups did not differ in their latencies to begin licking $[\mathrm{t}(6)=.17]$. However, in the presence of $S_{2}$, the tone, the latency from Lick 5 to Lick $\mathbf{4 0}$ was significantly greater in Group Same than in Group Different $[t(6)=2.61, p<.05$; see left panel of Figure 1].

These data demonstrate that the two conditioning contexts, as intended, were discriminably different to the subjects. Moreover, they support the hypothesis that a conditioned contextual stimulus can serve as a reinforcer for another stimulus. Following the pair-. ing of a context with a US, fear conditioning of a second stimulus is greater when $S_{2}$ is presented in the training context than when it is presented in a different context. The obtained difference is consistent with second-order conditioning, because it depended on the prior conditioning history of $S_{1}$ and on the paired presentation of $S_{2}$ and $S_{1}$.

\section{EXPERIMENT 2}

A potential alternative explanation of the results of Experiment 1 can be formulated with respect to the novelty of the context, $S_{1}$, during the second phase of second-order conditioning. Because Group Different heard the tone in a context that was different from that in which it had been given context- 


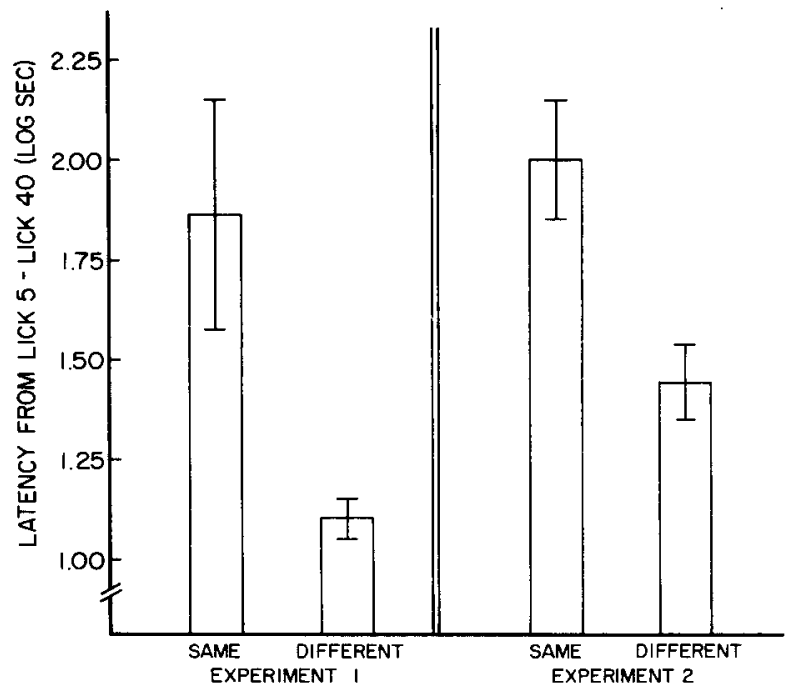

CONTEXTS IN WHICHFOOTSHOCKS AND TONES WERE PRESENTED

Figure 1. The mean and standard error of the test $\log$ (base 10) latencies from Lick 5 to Lick 40 in the presence of $S_{2}$ for groups in Experiment 1 (left panel) and Experiment 2 (right panel).

shock pairings, the context in which the tone was presented was novel. In contrast, the context during the second phase of second-order conditioning was not novel to subjects in Group Same because they had previously received shocks in that particular context. Thus, Group Different and Group Same differed not only in whether $S_{2}$ was paired or unpaired with $S_{1}$, but also in terms of whether $S_{1}$ was novel or familiar to the subjects during $S_{2}-S_{1}$ pairings. It is conceivable that this novelty confound may have been responsible for the results of Experiment 1the tone may have been differentially effective depending on the familiarity of the context (Lubow, Rifkin, \& Alex, 1976; Marlin \& Miller, 1981).

To address this issue, Experiment 2 modified the procedure of Experiment 1 to equate context exposure in both conditions. Essentially, during the first phase of second-order conditioning, each subject received three exposures to one of the contexts. During each of these exposures, footshocks were administered These context-footshock pairings alternated with nonreinforced exposures of equal duration to the other context. In this way, all subjects received equal exposure to both contexts to insure that neither context was novel during the second phase of secondorder conditioning.

\section{Method}

Subjects and Apparatus. Eight rats, similar to those described in Experiment 1, served as subjects. The apparatus was the same as that used in Experiment 1.

Procedure. Days 1 and 3, the lick exposures, were identical to the first experiment. On Day 2, each subject received three 2.5min exposures to one of the two training contexts; the actual context assigned and order of presentation of the contexts were counterbalanced across subjects. During each exposure, two .5 -sec, .5-mA constant-current footshocks were given with a variable $75-\mathrm{sec}$ intershock interval. Following each exposure to the training context, the subject was placed in the other context for $2.5 \mathrm{~min}$; no footshocks were presented during these context exposures.

Day 4 consisted of the second phase of second-order conditioning and the test of $S_{2}$. As in Experiment 1, the subjects assigned to each of the two training contexts were subdivided to form two groups. Group Same had $S_{2}$ paired with $S_{1}$, the training context; Group Different had $S_{2}$ paired with the other context, in which they had never received the US. In each case, the subject was placed in the appropriate context for $60 \mathrm{sec}$, during which it heard two 5-sec, 1600-Hz, 77-dBA tones.

Beginning $30 \mathrm{~min}$ after these $S_{2}$ presentations, the subject was placed in the lick apparatus. The tone began when the subject reached Lick 5 and remained on continuously until the subject completed Lick 40 . The latency to begin licking and the latency from Lick 5 to Lick $\mathbf{4 0}$ were recorded, using a ceiling score of $900 \mathrm{sec}$

\section{Results and Discussion}

On the measures of latency to begin licking on Days 1, 3, and 4, and on the total number of licks made on Days 1 and 3, Group Different and Group Same did not differ reliably (all ps $>.10$ ). Fear of $\mathrm{S}_{2}$, as measured by the latency from Lick 5 to Lick $\mathbf{4 0}$ on the lick suppression test, was significantly greater in Group Same than in Group Different $[t(6)=3.19$, $\mathrm{p}<.02$; see right panel of Figure 1].

This difference replicates the findings of Experiment 1 . The animals that had the tone paired with the same context that had been paired with footshock $48 \mathrm{~h}$ earlier demonstrated greater conditioned fear to the tone than did those animals that had received exposure to the same stimuli but for which the tone and the shock were paired with different contexts. Given the present procedure of alternating subjects between the reinforced and nonreinforced context during the first phase of second-order conditioning, it may be questioned whether the nonreinforced context may have acquired inhibitory properties for which the lick suppression measure was insensitive. It is generally observed, however, that for a stimulus to become a conditioned inhibitor of fear, it must occur in an excitatory context in which shocks are expected. Since the nonreinforced context in the present experiment was never paired with the footshock, and since the test data show that the subjects clearly did discriminate between the two contexts, the possibility of inhibitory contextual conditioning appears unlikely.

\section{EXPERIMENT 3}

The preceding experiments report that rats receiving a tonal stimulus in a context in which they had previously been shocked show greater conditioned fear of that stimulus than do rats that received the tone and the shocks in different contexts. This difference has been interpreted as supporting the hypothesis that a contextual stimulus can effectively function as $S_{1}$ in a second-order conditioning procedure. It 
should be noted, however, that it is possible to explain this difference in fear of the tone as a nonassociative effect of $S_{2}$, the tone, on lick suppression. To the extent that the presence of excitatory background cues interfered with the processing of $S_{2}$ and consequently protected $S_{2}$ against habituation, the lick-suppression data may reflect differential habituation between the treatment conditions rather than second-order conditioning. In order to control for this possibility, Experiment 3 included a US-only condition. This group received only the first phase, that is, $S_{1}$-US pairings, of second-order conditioning. If the present effect was due to differential habituation, then the unconditioned suppression to $S_{2}$ in this control group ought to be similar to the lick suppression of the group that received the tone in the context in which they had been shocked.

The present experiment also extended the procedure used in Experiment 2 by exposing all subjects to both the reinforced and nonreinforced contexts during each phase of second-order conditioning. This explicitly unpaired procedure assures that the conditioned responding to $S_{\mathbf{2}}$ in the experimental group is specific to the associative relationships between $S_{2}$ and $S_{1}$ and between $S_{1}$ and the US.

\section{Method}

Subjects and Apparatus. The subjects were 24 experimentally naive female rats weighing between 225 and $353 \mathrm{~g}$. They were maintained in the same way as were the rats in Experiments 1 and 2 . The apparatus was the same as that used in the preceding experiments.

Procedure. The subjects were divided randomly into three groups: Group Same, Group Different, and Group US Only $(n=8)$. All subjects were given lick exposures, as described in Experiment 1 , on Days 1 and 3. The first phase of second-order conditioning was given on Day 2, which was identical to Day 2 of Experiment 2 in that all subjects were alternately exposed to the reinforced and nonreinforced contexts. Context assignment and order of presentation were completely counterbalanced in each condition. On Day 4, subjects in Group Same and Group Different were given the second phase of second-order conditioning. During a 60 -sec exposure to either the training context (Group Same) or the context in which they had not been given the footshock (Group Different), two 5-sec presentations of the $1600-\mathrm{Hz}$, 77-dBA tone were given. Each subject in these two groups was also placed in the other context for $60 \mathrm{sec}$. No experimental treatment was given to Group US Only during this phase; they were never exposed to the tonal $S_{2}$ prior to the lick-suppression test.

Beginning $30 \mathrm{~min}$ later, each subject was tested for its lick suppression to $S_{2}$, using the test procedure described for Experiments 1 and 2.

\section{Results and Discussion}

One subject in Group US Only was removed from the study when it failed to lick during the lick-exposure session on Day 3; the Day 1 data for this subject was not included in the analysis. There were no differences among the three groups in their latencies to begin licking or in the number of licks made on Day 1 or Day 3 (all ps $>.10$ ). Similarly, there were no differences among the three groups in their latency to Lick 1 on the test $[F(2,20)=.52]$. In the presence of
$S_{2}$, reliable differences were obtained in their latencies from Lick 5 to Lick 40. Consistent with the results of Experiments 1 and 2, Group Same had a longer latency than Group Different $[t(14)=4.24$, $\mathrm{p}<.01]$. The lick suppression in Group Same was also significantly greater than that of Group US Only $[t(13)=3.18, p<.01]$, but the suppression of this latter group did not differ from that of Group Different $[t(13)=.89$; see Figure 2].

These data are inconsistent with the differential habituation hypothesis. Group US Only had never heard $S_{2}$ and therefore had no opportunity to habituate to it prior to the test trial. In spite of this, Group US Only had a significantly lower latency than did Group Same. Furthermore, given that the suppression of Group US Only did not differ from that of Group Different, it would appear that there are few or no unconditional suppressive effects of this relatively low-intensity tone on lick suppression. This finding, coupled with the explicitly unpaired presentation of the contexts in each phase of Experiment 3, provides further support for interpreting the present effect in terms of a second-order conditioning process.

\section{GENERAL DISCUSSION}

The results of these experiments suggest that following the pairing of a contextual stimulus with an unconditioned stimulus, the contextual stimulus acquires the ability to serve as a reinforcer for another stimulus in a second-order conditioning paradigm. That is, a sufficient procedure for second-order conditioning is the presentation of $S_{2}$ within a context

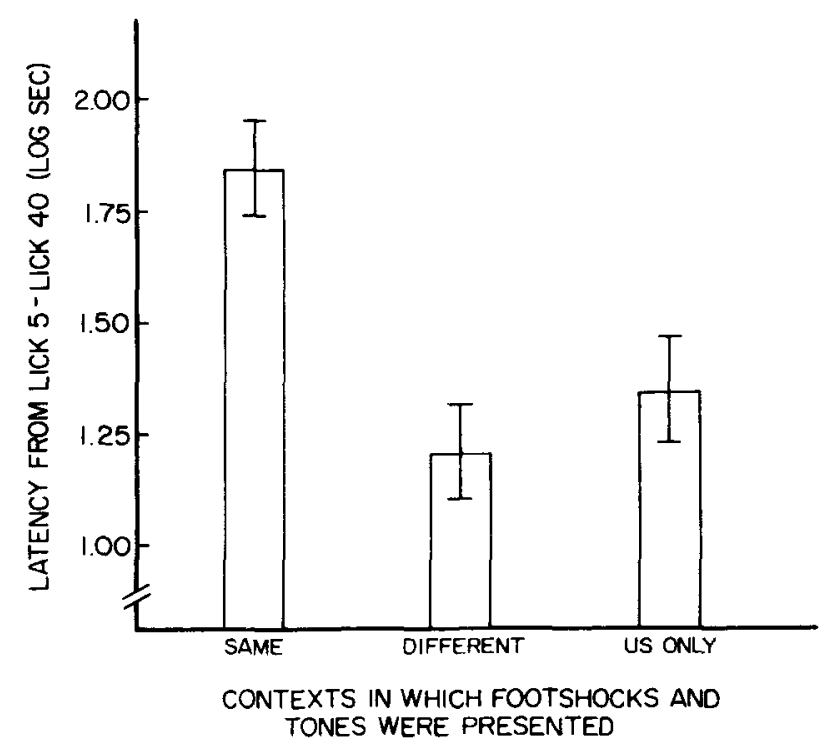

Figure 2. The mean and standard error of the test $\log$ (base 10) latencies from Lick 5 to Lick 40 in the presence of $S_{2}$ for groups in Experiment 3. 
that has previously been paired with a US. The present experimental designs demonstrated the associative specificity of the obtained conditioning and ruled out explanations of this effect in terms of first-order conditioning of $S_{2}$, differential generalization between the training context and the test context, context novelty at the time of the $S_{2}-S_{1}$ pairing, or differential habituation to $S_{2}$.

The finding that an external context, which is essentially a spatial stimulus, can serve as an $S_{1}$ in second-order conditioning is relevant for traditional control procedures in classical conditioning. The procedures that are most widely used, such as the explicitly unpaired or truly random control (see Mackintosh, 1974), involve presenting the CS and the US in the same context. This raises the possibility that any response observed to the CS in such control groups, rather than representing factors such as sensitization or fortuitous pairings, may result from second-order conditioning. This is not to question the appropriateness of such procedures, but to appreciate that they are designed to control for temporally mediated associative relationships. It is possible that these control procedures may yield spatially mediated associations through second-order conditioning with the context serving as $S_{1}$. For instance, Rizley and Rescorla (1972) conducted a second-order conditioning experiment in which one control group received tone-light pairings following explicitly unpaired presentations of the light and a footshock. On the first block of trials, conditioned responding to the tone in this control group was equal to that of the experimental group in which the stimuli were paired in both phases of second-order conditioning. This initially high level of responding to the tone was interpreted as first-order conditioning produced by some aversiveness of the light itself. However, since the light was presented in the same context as the footshocks, it may be that the light acquired its aversiveness through second-order conditioning. In contrast, many explicitly unpaired or truly random control groups used in conditioning procedures exhibit low levels of conditioned responding despite the presentation of both the CS and the US within the same experimental context. This may indicate that the use of a contextual stimulus in second-order conditioning requires few trials and relatively short exposures to the context. Such constraints are typical in secondorder conditioning, since they minimize the opportunity for $S_{1}$ to undergo extinction.

The present experiments demonstrated that secondorder conditioning can occur when a spatial stimulus serves as $S_{1}$. This finding extends the generality of this conditioning phenomenon, but it does not detract from the role of temporal contiguity in the formation of such associations. Unfortunately, these data provide little insight into the theoretical role of the context. It is unclear whether the context in this procedure is directly associated with the US during the first phase of second-order conditioning or whether it is able to influence behavior independently of any direct context-US associations (e.g., Bouton \& King, 1983; Nadel \& Willner, 1980). The investigation of second-order conditioning may prove beneficial in analyzing contextual associations in much the same way as it has in the analysis of associations between temporally discrete stimuli (Rescorla, 1980). Moreover, irrespective of the theoretical role of the context, the present procedure provides a new method for assessing the strength of contextual stimuli. Although the present experiments did not manipulate the amount of contextual conditioning, the associative value of the context would be predicted to influence its ability to serve as a reinforcer for a neutral stimulus.

\section{REFERENCES}

Balsam, P. D., \& Schwartz, A. L. Rapid contextual conditioning in autoshaping. Journal of Experimental Psychology: Animal Behavior Processes, 1981, 7, 332-393.

Best, P. J., Best, M. R., \& Mickley, G. A. Conditioned aversion to distinct environmental stimuli resulting from gastrointestinal distress. Journal of Comparative and Physiological Psychology, 1973, 85, 250-257.

Bouton, M. E., \& King, D. A. The contextual control of the extinction of conditioned fear: Tests for the associative value of the context. Journal of Experimental Psychology: Animal Behavior Processes, 1983, 9, 248-265.

Cunningham, C. L. Alcohol as a cue for extinction: State dependency produced by conditioned inhibition. Animal Learning \& Behavior, 1979, 7, 45-52.

Lubow, R. E., Rifkin, B., \& Alek, M. The context effect: The relationship between stimulus pre-exposure determines subsequent learning. Journal of Experimental Psychology: Animal Behavior Processes, 1976, 1, 38-47.

Mackintosh, N. J. The psychology of animal learning. New York: Academic Press, 1974.

Marlin, N. A. Contextual associations in trace conditioning. Animal Learning \& Behavior, 1981, 9, 519-523.

Marlin, N. A., \& Miller, R. R. Associations to contextual stimuli as a determinant of long-term habituation. Journal of EXperimental Psychology: Animal Behavior Processes, 1981, 7, 313-333.

NADEl, L., \& Willner, J. Context and conditioning: A place for space. Physiological Psychology, 1980, 8, 218-228.

Opling-SmeE, F. J. The overshadowing of background stimuli by an informative $\mathrm{CS}$ in aversive Pavlovian conditioning with rats. Animal Learning \& Behavior, 1978, 6, 43-51.

Rescorla, R. A. Pavlovian second-order conditioning. Hillsdale, N.J: Erlbaum, 1980.

Rescorla, R. A., \& Wagner, A. R. A theory of Pavlovian conditioning: Variations in the effectiveness of reinforcement and nonreinforcement. In A. H. Black \& W. F. Prokasy (Eds.), Classical conditioning II. New York: Appleton-Century-Crofts, 1972.

Rizley, R. C., \& Rescorla, R. A. Associations in second-order conditioning and sensory preconditioning. Journal of Comparative and Physiological Psychology, 1972, 81, 1-11.

Tomie, A., Murphy, A. L., FAth, S., \& Jackson, R. L. Retardation of autoshaping following pretraining with unpredictable food: Effects on changing the context between pretraining and testing. Learning \& Motivation, 1980, 11, 117-134.

(Manuscript received January 12, 1983; revision accepted for publication April 25, 1983.) 